\title{
\begin{tabular}{l|l} 
Mitraries & DSpace@MIT
\end{tabular}
}

\author{
MIT Open Access Articles
}

\section{Passive Prosthetic Foot Shape and Size Optimization Using Lower Leg Trajectory Error}

The MIT Faculty has made this article openly available. Please share how this access benefits you. Your story matters.

Citation: Olesnavage, Kathryn M., and Amos G. Winter. "Passive Prosthetic Foot Shape and Size Optimization Using Lower Leg Trajectory Error." Volume 5A: 41st Mechanisms and Robotics Conference (August 6, 2017).

As Published: http://dx.doi.org/10.1115/DETC2017-67618

Publisher: ASME International

Persistent URL: http://hdl.handle.net/1721.1/120850

Version: Final published version: final published article, as it appeared in a journal, conference proceedings, or other formally published context

Terms of Use: Article is made available in accordance with the publisher's policy and may be subject to US copyright law. Please refer to the publisher's site for terms of use. 


\title{
PASSIVE PROSTHETIC FOOT SHAPE AND SIZE OPTIMIZATION USING LOWER LEG TRAJECTORY ERROR
}

\author{
Kathryn M. Olesnavage* \\ Amos G. Winter, V \\ Global Engineering and Research (GEAR) Laboratory \\ Department of Mechanical Engineering \\ Massachusetts Institute of Technology \\ Cambridge, MA 02139 \\ Email: kolesnav@mit.edu
}

\section{ABSTRACT}

A method is presented to optimize the shape and size of a passive prosthetic foot using the Lower Leg Trajectory Error (LLTE) as the design objective. The LLTE is defined as the rootmean-square error between the lower leg trajectory calculated for a given prosthetic foot by finding the deformed shape of the foot under typical ground reaction forces and a target physiological lower leg trajectory obtained from published gait data for able-bodied walking. In previous work, the design of simple two degree-of-freedom analytical models consisting of rigid structures, rotational joints with constant stiffness, and uniform cantilevered beams, have been optimized for LLTE. However, prototypes built to replicate these simple models were large, heavy, and overly complex. In this work, the size and shape of a singlepart compliant prosthetic foot keel made out of nylon $6 / 6$ was optimized for LLTE to produce a light weight, low cost, and easily manufacturable prosthetic foot design. The shape of the keel was parameterized as a wide Bézier curve, with constraints ensuring that only physically meaningful shapes were considered. The LLTE value for each design was evaluated using a custom MATLAB script, which ran ADINA finite element analysis software to find the deformed shape of the prosthetic keel under multiple loading scenarios. The optimization was performed by MATLAB's built-in genetic algorithm. After the optimal design for the keel was found, a heel was added to structure, sized such

\footnotetext{
*Address all correspondence to this author.
}

that when the user's full weight acted on the heel, the structure had a factor of safety of two. The resulting optimal design has a lower LLTE value than the two degree-of-freedom analytical models, at 0.154 compared to 0.172, 0.187, and 0.269 for the two degree-of-freedom models. At $412 \mathrm{~g}$, the optimal wide curve foot is nearly half the mass of the lightest prototype built from the previous models, which was $980 \mathrm{~g}$. The design found through this compliant mechanism optimization method is thus far superior to the two degree-of-freedom models previously considered.

\section{INTRODUCTION}

Numerous studies have shown that the mechanical design of a passive prosthetic foot affects the users' gait [1-8]. However, there is no consensus on exactly how the mechanical properties of a foot relate to the biomechanical performance of the foot [912]. Without this relationship, it is impossible to optimize the design of a prosthetic foot for peak performance, or to evaluate potential tradeoffs when designing low cost feet for emerging markets with minimal sacrifice of performance.

One proposed design objective for prosthetic feet is to replicate physiological roll-over geometries. The roll-over geometry of a foot is defined as the path of the center of pressure along the bottom of the foot from heel strike to opposite heel strike as measured in the ankle-knee reference frame [13]. It has been shown that roll-over geometries are fairly consistent for individu- 
als with similar leg lengths, and that roll-over geometries remain unchanged through variations in walking speed, shoe heel height, and carried torso weight [14-16]. Because the roll-over geometry can be measured both mechanically for a prosthetic foot without a human subject that inherently introduces variability and for typical, unimpaired walking for biological feet, it provides a connection between the mechanical properties of the foot and gait mechanics that can be utilized to optimize prosthetic feet to better replicate physiological functionality. However, the roll-over geometry is measured only in the ankle-knee reference frame and does not include any information about the orientation of this reference frame relative to the global reference frame. Thus it does not fully describe the functionality of a given prosthetic foot. Previous work has demonstrated that it is possible for two different prosthetic feet to have identical roll-over geometries but yield very different lower leg kinematics under the same ground reaction forces (GRFs) [17,18]. Therefore, roll-over geometry is insufficient to be used in optimizing the design of prosthetic feet, as ideal kinematics cannot be ensured.

The authors have developed a novel design objective, called the Lower Leg Trajectory Error (LLTE), that quantifies how closely the position of the lower leg segment for a given prosthetic foot is able to replicate target physiological lower leg positions throughout the course of a step $[17,18]$. Three simple prosthetic foot architectures, each with two degrees of freedom, have previously been optimized using LLTE to demonstrate its usefulness as a design tool. The first model was a rigid circular foot, with the radius of the circle and the horizontal position of the center of the circle as design variables. The second consisted of rotational pin joints at the ankle and metatarsal joints, with the rotational stiffness of each joint as the design variables. The third and final model considered also consisted of a rotational ankle joint, but replaced the metarsal joint with a compliant cantilever beam forefoot, with the ankle stiffness and the forefoot beam bending stiffness as design variables. Multiple prototypes based on these simple architectures have been built and are being used in clinical testing to validate the LLTE optimization method, with promising initial results $[19,20]$. However, while the simple, two degree-of-freedom, architectures have been useful tools to rapidly iterate through experimental prototypes and effectively proove the concept of prosthetic foot optimization based on LLTE, the resulting prototypes are large, heavy, and consist of relatively complex mechanisms. In order to translate these experimental prototypes to commercial products, a lighter, more robust, and easier to manufacture design is required.

The goal of this work was to apply compliant mechanism optimization techniques to design a single-part foot that minimizes the LLTE to best replicate physiological lower leg kinematics. The design space parametrization, based on a wide Bézier curve, is discussed, together with constraints that were applied to ensure only physically-meaningful shapes were considered. The evaluation of the LLTE value for a given design using MATLAB and
ADINA finite element analysis software is elucidated. The optimal design is presented and compared to the simple analytical models previously optimized.

\section{Lower Leg Trajectory Error}

LLTE is defined as

$$
\begin{aligned}
& \operatorname{LLTE} \equiv\left[\frac { 1 } { N } \sum _ { n = 1 } ^ { N } \left\{\left(\frac{x_{n}-\hat{x}_{n}}{\hat{\bar{x}}}\right)^{2}\right.\right. \\
&\left.\left.+\left(\frac{y_{n}-\hat{y}_{n}}{\hat{\bar{y}}}\right)^{2}+\left(\frac{\theta_{n}-\hat{\theta}_{n}}{\hat{\bar{\theta}}}\right)^{2}\right\}\right]^{\frac{1}{2}},
\end{aligned}
$$

where $x_{n}$ and $y_{n}$ are the horizontal and vertical positions of the knee and $\theta_{n}$ is the orientation of the lower leg segment with respect to vertical, as calculated for a given prosthesis under an assumed set of ground reaction force and center of pressure data at the $n^{\text {th }}$ time interval, where stance phase is divided into a total of $N$ intervals. The variables $\hat{x}_{n}, \hat{y}_{n}$, and $\hat{\theta}_{n}$ refer to the same values as measured for target able-bodied walking, and $\hat{\bar{x}}, \hat{\bar{y}}$, and $\hat{\bar{\theta}}$ are the mean physiological values over all $N$ time intervals, which serve to normalize the errors in each variable. Throughout this work, all physiological gait data comes from Winter's published data, which were obtained from a subject of body mass $56.7 \mathrm{~kg}[21]$.

\section{Compliant Mechanism Optimization}

The goal of this work was to move away from the bulky articulated rigid body mechanisms previously analyzed and instead design a prosthetic foot structure consting of a single part that, in response to specific loading scenarios, deforms elastically in such a way as to achieve a desired output motion, that is, a compliant mechanism [22]. There is a plethora of literature on topology synthesis and optimization for compliant mechanisms [23-29] including continuum element density approaches, frame element-based structures, and pseudo-rigid body models. However, the outputs of these topology optimizations have several practical limitations; for example, they consist only of uniform elements or uniform cross-sections, have unclear boundaries or checkerboard patterns, or they result in localized flexural hinges with high stress concentrations [30]. Furthermore, the topology of a prosthetic foot does not need to be complicated. All that is required is material at the ankle that can be attached to the rest of the prosthesis, and a flat bottom surface of the foot upon which the center of pressure can progress smoothly from heel-strike to toe-off. What remains to be optimized is only the size and shape of the mechanism connecting the ankle to the bottom of the foot. 
Several methods for compliant mechanism size and shape optimization were considered [31-33], but ultimately the foot was approached using a wide Bézier curve, as presented by Zhou and Ting [34]. A wide Bézier curve is a parametric curve with a shape dictated by a series of control points. With a Bézier curve, a cubic curve can be defined by the position of four control points, reducing a potentially complex shape to a limited number of design variables. The width is added as a variable by using control circles rather than control points and defining the width of the wide curve as a function of the diameters of these control circles. The output of an optimization method such as this is a $2 \mathrm{D}$ shape that is easily manufacturable with minimal postprocessing, which means the theoretical optimization result can quickly be built as a physical prototype.

\section{METHOD}

\section{Size and Shape Parameterization}

The goal of this optimization problem was to design a single part compliant foot that, when acted upon by typical ground reaction forces, deformed in such a way as to best replicate typical lower leg kinematics, as quantified by minimizing the Lower Leg Trajectory Error. For the scope of this work, only the design of the forefoot was optimized, as many prosthetic feet de-couple early stance from the rest of stance phase by using a separate mechanism, such as a cushion or a secondary compliant mechanism, for the heel portion of the foot. After the optimal forefoot was found, a heel was added to the forefoot structure and sized appropriately such that it could withstand the full mass of the user with a factor of safety of two.

The shape and size of the forefoot was defined by four control points $\left(C_{1}, C_{2}, C_{3}\right.$ and $C_{4}$ in Fig. 1), each of which had an $\mathrm{x}$-position, y-position, and a diameter, denoted by subscripts $x$, $y$, and $d$, respectively. The first node, $C_{1}$, was the point of attachment between the foot and the rest of the prosthesis, and was fixed at $\left(C_{1 x}, C_{1 y}\right)=(0,0)$. Throughout the course of this work, all measurements and coordinates are in units of meters, unless otherwise stated. The height of the foot from the attachment point to the bottom of the foot was $h$, such that $C_{4 y}=-h+\frac{1}{2} C_{4 d}$, where $C_{4 d}$ was the width of the foot at $C_{4}$. To prevent any kinks in the structure, the tangent to the Bézier curve at point $C_{4}$ was made horizontal by enforcing $C_{3 y}=C_{4 y}$. The coordinate $C_{4 x}$ was defined by the horizontal position of the center of pressure at the first instant in Winter's published gait data for which the center of pressure was proximal to the ankle in the ankle-knee reference frame, that is, $C_{4 x}=0.02 \mathrm{~m}$. The foot extended forward from $C_{4}$ to the tip of the foot, $C_{5}$, with $C_{5 x}=0.15 \mathrm{~m}$. The width of the forefoot decreased linearly from $C_{4}$ to the tip of the foot, with the design variable $f f_{\text {frac }}$ defining the ratio of the width of the tip of the forefoot to the width of the foot at $C_{4}$. That is, $f f_{\text {frac }}=C_{5 d} / C_{4 d}$. In order to keep the foot flat and stable on the ground when it was unloaded, $C_{5 y}=-h+\frac{1}{2} f f_{f r a c} \cdot C_{4 d}$. Thus there were nine independent design variables to be optimized:

$$
X=\left[h, C_{1 d}, C_{2 x}, C_{2 y}, C_{2 d}, C_{3 x}, C_{3 d}, C_{4 d}, f f_{\text {frac }}\right]
$$

Upper and lower bounds were imposed on each of the variables as

$l b=[0.06,0.005,-0.07,-0.10,0.005,-0.07,0.005,0.005,0.1]$

and

$$
u b=[0.15,0.04,0.07,0.10,0.04,0.01,0.04,0.04,1]
$$

to constrain the shape and size of the structure to approximately fit within the envelope of a biological foot. The thickness of the foot into the plane was fixed at $0.06 \mathrm{~m}$ such that the foot can easily fit into a shoe or cosmesis.

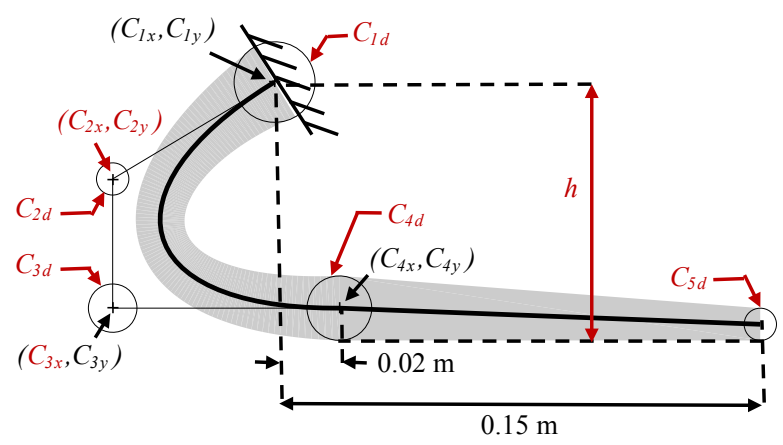

FIGURE 1: Parameterization of the keel of the foot. The shape and size of the keel are defined with nine independent design variables, shown here in red.

Examples of possible foot shapes explored through this particular parametrization are shown in Fig. 2.

\section{Materials}

The optimization was performed using nylon 6/6, with elastic modulus $E=2.41 \mathrm{GPa}$ and yield strength $\sigma_{y}=82.7 \mathrm{MPa}$. Nylon was selected as a reasonable material choice for a low cost prosthetic foot because the high ratio of yield strength to elastic modulus allows nylon to achieve high deformations before yielding. 

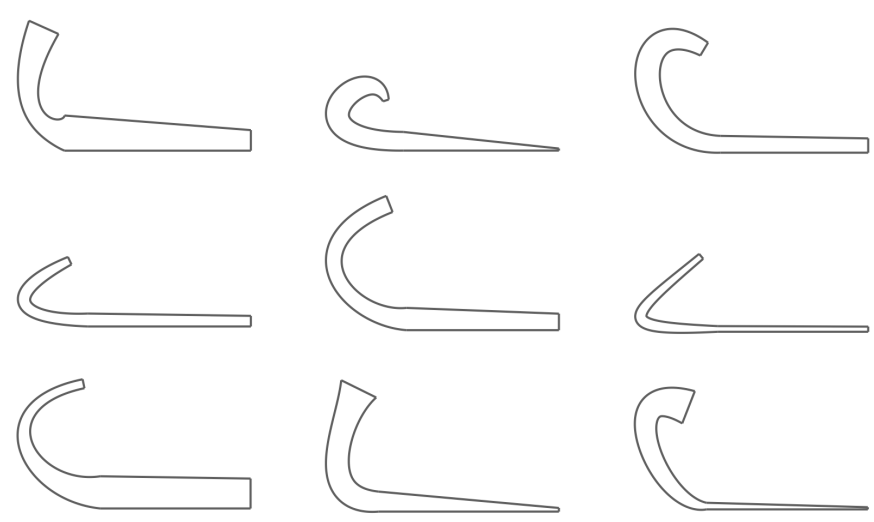

FIGURE 2: Various possible keel designs that fall within the defined design space

\section{Constraints}

It is possible that a particular set of design variables could yield a wide Bézier curve that intersects itself, resulting in a shape with no physical meaning. Self-intersection occurs either when the radius of curvature of the center Bézier curve is less than half the width of the outer shape (Fig. 3a), or the center curve creates a loop (Fig. 3b). These self-intersections can be prevented with the following constraints:

$$
\max \left(0.5 w_{c}-\rho\right) \leq 0
$$

and

$$
\left(\frac{l \overline{C_{1} C_{2}}}{l \overline{Q C_{1}}}-\frac{4}{3}\right)\left(\frac{l \overline{C_{2} C_{3}}}{l \overline{Q C_{2}}}-\frac{4}{3}\right)-\frac{4}{9} \leq 0
$$

where $\rho$ is the radius of curvature of the center Bézier Curve, $Q$ the point of intersection of line segments $\bar{C}_{1} C_{2}$ and $\bar{C}_{3} C_{4}$, as shown in Fig. $3 \mathrm{~b}$, and $l \overline{C_{1} C_{2}}$ is the length of the line segment between control points $C_{1}$ and $C_{2}$ and so on.

Since $C_{3 y}=C_{4 y}$ and the shape has been defined such that the bottom of the control circle $C_{4}$ is the bottom of the foot, if $C_{3} d$ were greater than $C_{4 d}$ then the foot would protrude below the intended bottom surface. Therefore the linear inequality constraint

$$
C_{3 d}-C_{4 d} \leq 0
$$

was imposed.

Finally, a constraint was imposed to limit the maximum stress in the foot structure: (a) Self-Intersection Constraint Violation

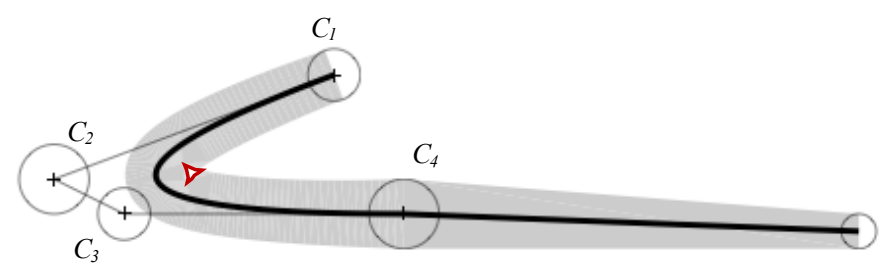

(b) Loop Constraint Violation

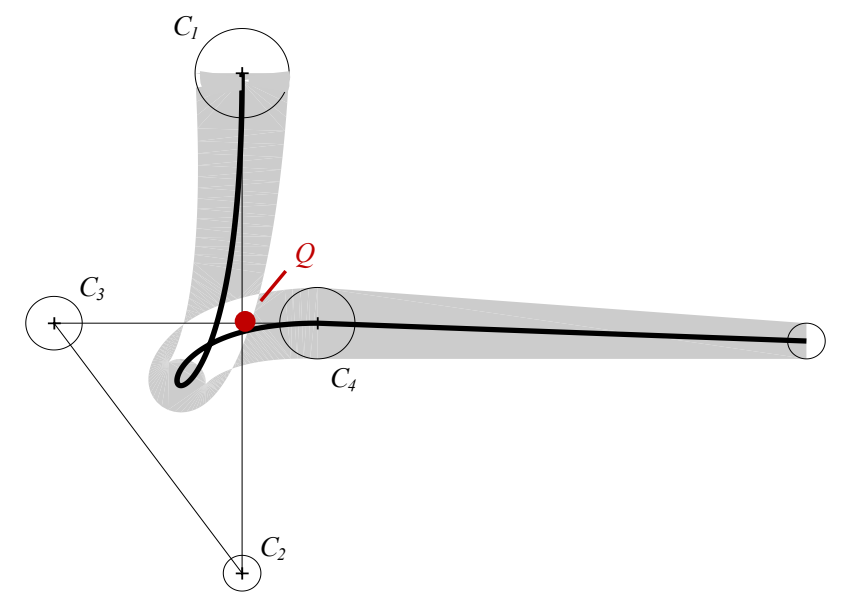

FIGURE 3: Certain combinations of design variables may result in the keel shape intersecting itself, creating a design that is not physically meaningful. Constraints are imposed to prevent cases like those shown here from being included in the optimization.

$$
\sigma_{\text {max }}-\sigma_{\text {allow }} \leq 0
$$

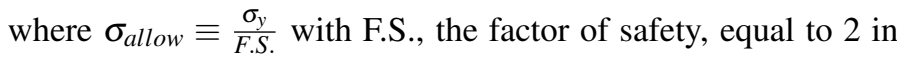
this case. The maximum stress in the structure, $\sigma_{\max }$, was found through the finite element solution.

\section{Evaluating LLTE}

For the simple foot architectures previously studied to demonstrate the usefulness of LLTE, the deformation of the foot under a given load, and consequently the $x, y$, and $\theta$ coordinates of the lower leg segment, could be calculated analytically. Thus each $x_{n}, y_{n}$, and $\theta_{n}$ calculation was inexpensve, so it was possible to find these values for every time interval during a step for which data were obtained. Using Winter's published data set and only considering the portion of stance for which the ankle angle is less than $90^{\circ}$, there are a maximum of $N=26$ time intervals 
for which data is available, all of which were used in calculating the LLTE during optimization of the simple architectures.

For the shapes of prosthetic feet considered in this work, there is no analytical solution to find the deformation of the foot structure in response to a given load. Rather, finite element analysis is required. To evaluate the LLTE for a single design, FEA must be performed $N$ times to calculate the deformation at each of the $N$ time intervals. Since FEA is computationally expensive, it is advantageous to minimize the number of time intervals required. To determine how many time intervals were necessary and which instances during the step were the best representation of the step as a whole, the LLTE optimization was performed for the simple analytical prosthetic foot models using each possible subset of the 26 total data points. It was found that with $N=5$, the optimal design variable values are each within $5 \%$ of those values found using all 26 data points if the five data points used are $33 \%, 48 \%, 60 \%, 74 \%$, and $81 \%$ of stance, where $0 \%$ is heel strike, $24 \%$ is the instant at which the ankle begins to dorsiflex past a neutral position, and $100 \%$ is toe-off (Fig. 4).

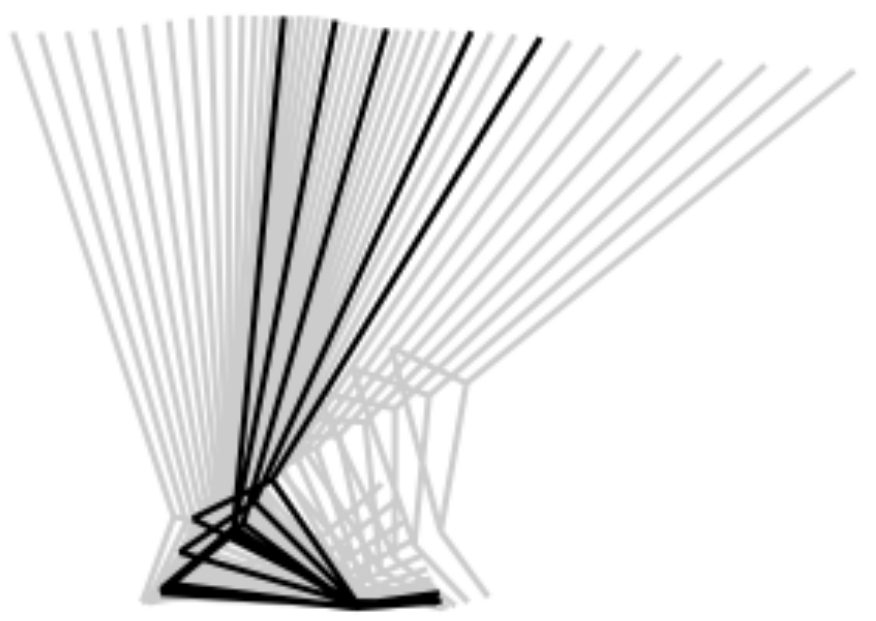

FIGURE 4: Of the 43 time intervals during stance included in Winter's published gait data shown in grey [21], the foot is flat on the ground and the ankle is in dorsiflexion for 26. Of those 26 , the five shown in black were found to best represent the entire step. When these five data points were used, the optimal design variable values for each of the two degree-of-freedom analytical models were each within a maximum difference of $5 \%$ of the optimal design variable values as found when all 26 available data points were used.

As an example, the ground reaction forces and the target positions of the lower leg segment for three of these five time intervals are as shown in Fig. 5. (a) Global Reference Frame
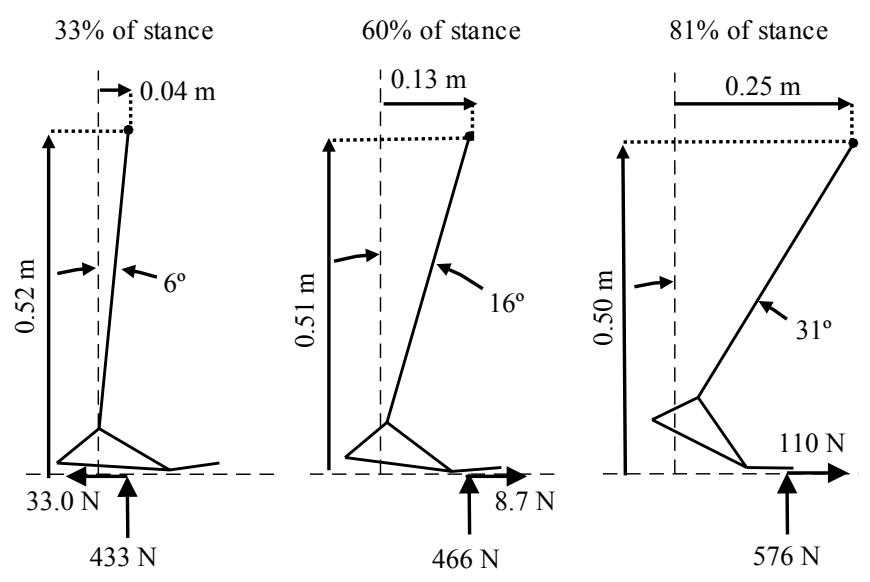

(b) Ankle-Knee Reference Frame
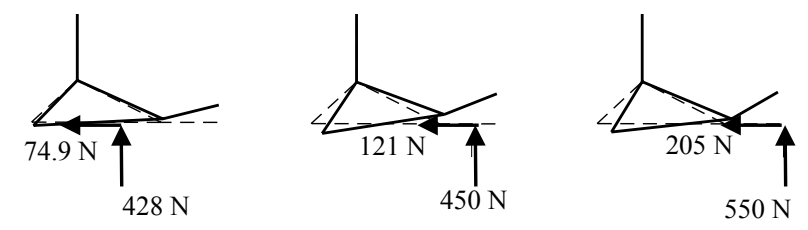

FIGURE 5: Free body diagrams of the ground reaction forces on the feet and the lower leg position during three of the five time intervals used in the finite element LLTE evaluation.

For a given set of design variables, $X$, FEA was performed on the foot five times, once for each of the five time intervals.

The $x, y$, and $\theta$ coordinates of the knee and lower leg segment can be found from just the position of the node at which the GRFs were applied, given by $\left(x_{\text {load }}, y_{\text {load }}\right)$ and the position of a node at the tip of the foot, $\left(x_{\text {end }}, y_{\text {end }}\right)$. Because the toe of the foot was unconstrained and the only external loads are the ground reaction forces, there are no internal bending moments within the foot structure between the point at which the GRFs are applied and the tip of the foot. Consequently, this portion of the foot is undeformed, and the bottom of the foot distal to the loading point remains straight. Then, for the center of pressure between the foot and the ground to indeed be at the node to which the loads have been applied, this entire segment of the foot, between the load point and the end of the foot, must be flat on the ground. The angle between the ground and the horizontal in the ankle-knee reference frame in which the FEA was performed, and, equivalently, the angle of the lower leg segment with respect to vertical in the global reference frame, was calculated from the FEA results as

$$
\theta=\tan ^{-1}\left(\frac{y_{\text {end }}-y_{\text {load }}}{x_{\text {end }}-x_{\text {load }}}\right)
$$


In the global reference frame, the origin was defined as the point of intersection between the ankle-knee axis and the ground when the ankle-knee axis is perpendicular to the ground during stance. Because the center of pressure data used as an input to the model is measured in the global reference frame, the $\mathrm{x}$ coordinate of the center of pressure in the global reference frame is the distance between the center of pressure and the origin of the global reference frame along the ground. Then the coordinates of the global origin in the ankle-knee reference frame, $x_{O, A K}$ and $y_{O, A K}$ are given by

$$
\left[\begin{array}{l}
x_{O, A K} \\
y_{O, A K}
\end{array}\right]=\left[\begin{array}{l}
x_{\text {load }}-x_{c p} \cos \theta \\
y_{\text {load }}-x_{c p} \sin \theta
\end{array}\right] .
$$

Finally, the position of the knee in the global reference frame was found by taking the vector from the global reference frame origin to the knee in the global reference frame, then rotating the vector by $\theta$. That is,

$$
\left[\begin{array}{l}
x \\
y
\end{array}\right]=\left[\begin{array}{cc}
\cos \theta & \sin \theta \\
-\sin \theta & \cos \theta
\end{array}\right] \cdot\left[\begin{array}{l}
x_{A K}-x_{O, A K} \\
y_{A K}-y_{O, A K}
\end{array}\right],
$$

where $x_{A K}$ and $y_{A K}$ are the coordinates of the knee in the ankle knee reference frame, so $x_{A K}=0$ and $y_{A K}=L_{A K}$, with $L_{A K}$ the length of the shank between the ankle and the knee, which comes from the distance from the knee to the ground in the input physiological data set minus the height of the prosthetic foot, $h$, for the particular design in consideration.

To automate the LLTE calculation for a particular design to allow for optimization, a custom MATLAB script was used to write and save text files containing input batch commands for ADINA, the commercially available FEA software used in this optimization. The commands within the text files defined the foot geometry as a 2D plane stress solid, meshed the surfaces, defined the material properties, and applied the appropriate loads. A boundary condition was applied at the ankle to fix all degrees of freedom, as the analysis was performed in the ankle-knee reference frame, so any external loads would be opposed by reaction forces and moments at the ankle point, where the prosthetic foot would connect to the rest of the prosthesis. The finite element analysis was run via command line prompts executed through MATLAB. The results, namely the deformed position of the load node and the end node, were saved in another text file, which was read and processed via another custom MATLAB script, which calculated the $x_{n}, y_{n}$, and $\theta_{n}$ corresponding to that load case using Equations (9) - (11). This was repeated for the other four load cases. Finally, the $x_{n}, y_{n}$, and $\theta_{n}$ and the target physiological $\hat{x}_{n}, \hat{y}_{n}$, and $\hat{\theta}_{n}$ values for all five cases were used with Eqn. (1) to calculate the LLTE value for that set of design variables.

\section{Optimization Problem Formulation}

The optimization problem to be solved was then:

$$
\left.\begin{array}{cl}
\min _{X}: & \operatorname{LLTE}(X) \\
\text { subject to: } \quad & \max \left(0.5 w_{c}-\rho\right) \leq 0 \\
: & \left(\frac{l \overline{C_{1} C_{2}}}{l \overline{Q C_{1}}}-\frac{4}{3}\right)\left(\frac{l \overline{C_{2} C_{3}}}{l \overline{Q C_{2}}}-\frac{4}{3}\right)-\frac{4}{9} \leq 0 \\
: & C_{3 d}-C_{4 d} \leq 0 \\
: & \sigma_{\text {max }}-\sigma_{\text {allow }} \leq 0
\end{array}\right\}
$$

The optimization was performed using MATLAB's built-in genetic algorithm function with a custom objective script which returned the LLTE value of a particular design following the previously described method.

\section{RESULTS}

The optimal design variable values resulting from the optimization were

$$
\begin{aligned}
X=[0.1461,0.0142,-0.0698,-0.0455 \\
\quad 0.0202,-0.0690,0.0156,0.0170,0.1031]
\end{aligned}
$$

with LLTE value 0.154, (Fig. 6). The maximum stress in the optimal nylon structure was 39.9 MPa for a safety factor of 2.07.

\section{DISCUSSION}

To contextualize the optimal design from this wide curve optimization, the LLTE optimal designs for the simple foot architectures previously presented found using only the same five loading scenarios that were used in the wide curve optimization, the minimum LLTE values are 0.269 for the rigid circular foot, 0.172 for the foot with the rotational ankle and metatarsal joints, and 0.187 for the foot with a rotational ankle joint and a cantilever beam forefoot. Since the LLTE value for the optimal wide curve foot is less than each of these, it comes closer to replicating the physiological lower leg trajectory across the five loading scenarios used. Additionally, because the wide curve design does not require multiple parts such as a spring, axis of rotation, or rigid structural elements, it can be made significantly lighter than either of the articulated simple architectures presented. The area of the optimal 2D shape with the heel is $60.8 \mathrm{~cm}^{2}$. Given the thickness into the plane, $6.0 \mathrm{~cm}$, and assuming a density of 


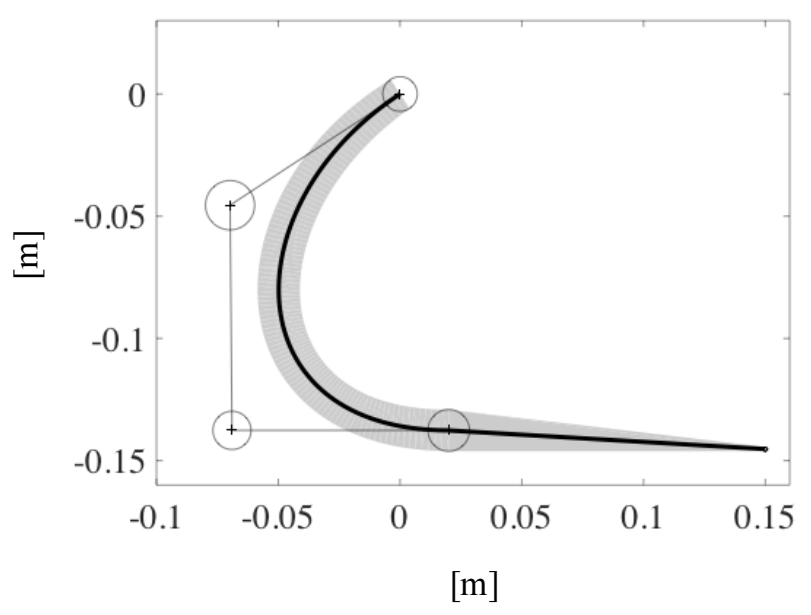

FIGURE 6: Optimal keel design found through the wide curve optimization method

$1.13 \mathrm{~g} / \mathrm{cm}^{3}$ for nylon, the resulting mass of the structure is $412 \mathrm{~g}$. Multiple experimental prototypes have been made to replicate the optimal designs of the simple architectures while minimizing the mass of the foot, but the minimum mass achieved was $980 \mathrm{~g}$, about twice the mass of the wide curve foot. Furthermore, the method presented here yields a design that is much easier to manufacture than the prototypes with articulated ankle joints, as the wide curve foot consists of a single nylon part that could easily be injection molded.

To build a foot from this theoretical design, all that is needed is a method to attach the foot to the rest of the prosthesis. The only boundary condition that must be met is that the ankle is fully fixed to the prosthetic pylon or prosthetic socket. This can be achieved simply by adding material to the ankle portion of the wide curve foot such that a standard male pyramid adapter can be bolted to the top (Fig. 7). Because this added structure is effectively rigid relative to the rest of the foot, the LLTE analysis remains unchanged.

The optimization took 14 hours, $18 \mathrm{~min}$ and 57 seconds to run, during which time the LLTE function was evaluated 6800 times for an average time of 7.6 seconds for a single design evaluation. The primary purpose of this work was to demonstrate that it is possible to use wide curve parameterization and a combination of MATLAB scripts and ADINA FEA software to produce a single-part prosthetic foot with minimal LLTE value. There are several limitations to this work that affect the general applicability of the resulting optimal foot that can be resolved easily, but will increase the optimization run time and thus were not addressed here. Before a foot is built and tested to clinically validate this work, further analyses will be performed to determine which of these limitations impact the results significantly enough

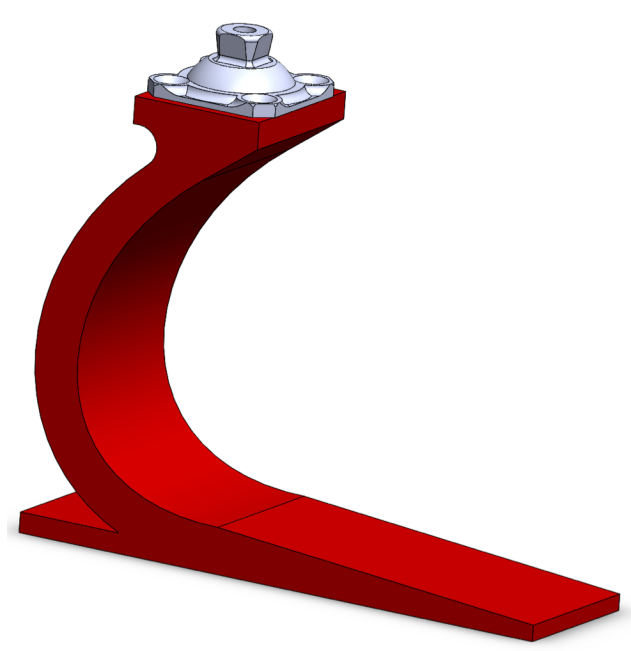

FIGURE 7: Solid model of foot based on optimal design, with added heel and male pyramid adapter to attach the foot to the rest of the prosthesis

to merit the additional optimization time that would be required to resolve them.

While an infinite number of possible designs were searched by this optimization problem, the complexity of the final design was limited by the definition of the design space, as was shown in Fig. 1. In future work, a more comprehensive design space will be explored by adding complexity with additional design variables, such as using higher-order Bézier curves to define the shape of the foot. The heel could also be incorporated into the optimization rather than optimizing the keel and forefoot and then designing a heel around that structure. Loading scenarios from early stance plantarflexion, when the center of pressure is posterior to the ankle, could then be included in the LLTE evaluation.

The shape of the foot has been optimized based on only five loading scenarios that are assumed to be adequately representative of the entire step. The lower leg trajectory of the prosthetic foot designed through the optimization may better replicate physiological gait kinematics throughout the whole step if more loading scenarios are included. The optimization runtime should scale linearly with the number of loading scenarios included, as each LLTE evaluation would perform an additional FEA simulation for each additional loading scenario, and the LLTE evaluation time is dominated by the FEA simulations. The number of function evaluations would not change significantly so long as the rate of convergence was not affected by the number of loading scenarios. Future work may including using more than five loading scenarios to determine if and how much the optimal design is affected.

Another potential source of error that could limit how accurately the FEA represents the kinematics of the foot during actual use is the direction of the GRF applied on the FE model. 
The input GRFs were measured in the global reference frame, then translated into the ankle-knee reference frame based on the orientation of the lower leg in the physiological data set to be applied to the ankle-knee reference frame-based FE model. The orientation of the ankle-knee reference frame of the wide curve foot during a particular load scenario depends on the deformed shape of the foot, which is dependent on the direction of the applied load. Thus, if and only if the foot deforms in such a way as to exactly replicate the orientation of the ankle-knee reference frame in the physiological data set, that is, $\theta_{n}-\hat{\theta}_{n}=0$ in Eqn.(1), the loading in the FEA is exactly equivalent to that in the input physiological gait data when both are rotated back into the global reference frame. Otherwise, the GRF magnitude is equivalent, but it is rotated by an amount equal to $\theta_{n}-\hat{\theta}_{n}$ relative to the GRF as measured in the global reference frame. For the optimal wide curve foot design presented here, the loading was rotated by a maximum of $1.41^{\circ}$ relative to the direction of the GRF measured in the global reference frame. This source of error could be eliminated through iteratively solving for the orientation of the ankle-knee reference frame for the wide curve foot. This iterative process would have to be repeated for each loading scenario, and each iteration would require an additional FEA simulation until the orientation of the ankle-knee reference frame used to calculate the loads applied to the FE model converges with the ankle-knee reference frame found based on the resulting deformed shape of the foot. This would consequently significantly increase the runtime of the LLTE evaluation for a single design, but would probably not affect the number of evaluations required for the optimization.

Because a set of published gait data for a single person was used both for the input kinetic data and for the target kinematic data, the optimal design is valid only for people of similar body mass and leg lengths as the subject with whom the data was recorded. After preliminary testing on subjects of similar size to clinically validate the method presented here, the method can easily be applied using sets of gait data for various body masses and leg lengths to produce a range of prosthetic feet to accomodate all potential users. Further, because it is easy to adjust the input data, future work may also involve customizing 3D printed prosthetic feet for specific individuals.

\section{CONCLUSION}

The shape and size of a prosthetic foot has been optimized as a compliant mechanism with the objective of minimizing the Lower Leg Trajectory Error (LLTE). The forefoot was parameterized as a wide Bézier curve with constraints imposed such that only physically meaningful shapes were considered. The deformed shape of each foot design was calculated for five different loading scenarios representative of different phases of stance using ADINA finite element analysis software, run through a custom MATLAB script. From the deformed shape of the foot, the position of the knee and the orientation of the lower leg segment were found and used to evaluate the LLTE for that particular design. The genetic algorithm built in to the MATLAB optimization toolbox performed the optimization. The optimal design was far superior to previously considered analytical prosthetic foot models with only two degrees-of-freedom, with an LLTE value for the compliant foot of 0.154 , which is better than the best of the analytical models at 0.172 , but roughly half the mass $(412 \mathrm{~g}$ for the compliant foot compared to $980 \mathrm{~g}$ for the lightest prototype based on the analytical models) and, with only a single injection-moldable part, far easier to manufacture. The resulting optimal design will be built and clinically tested in the near future.

\section{ACKNOWLEDGMENT}

This work was made possible by funding from the MIT Tata Center for Technology and Design. The authors would also like to thank Dr. Caroline Hargrove and her team at McLaren Applied Technologies, as well as Victor Prost, for all of their support.

\section{REFERENCES}

[1] Nederhand, M. J., Van Asseldonk, E. H., van der Kooij, H., and Rietman, H. S., 2012. "Dynamic Balance Control (DBC) in lower leg amputee subjects; contribution of the regulatory activity of the prosthesis side". Clinical Biomechanics, 27(1), pp. 40-45.

[2] Klodd, E., Hansen, A., Fatone, S., Hons, B. P. O., and Edwards, M., 2010. "Effects of prosthetic foot forefoot flexibility on gait of unilateral transtibial prosthesis users". Journal of Rehabilitation Research \& Development, 47(9), pp. 899-910.

[3] Klodd, E., Hansen, A., Fatone, S., and Edwards, M., 2010. "Effects of prosthetic foot forefoot flexibility on oxygen cost and subjective preference rankings of unilateral transtibial prosthesis users". Journal of Rehabilitation Research \& Development, 47(6), pp. 543-552.

[4] Bonnet, X., Villa, C., Fode, P., Lavaste, F., and Pillet, H., 2014. "Mechanical work performed by individual limbs of transfemoral amputees during step-to-step transitions: Effect of walking velocity". Proceedings of the Institution of Mechanical Engineers. Part H, Journal of engineering in medicine, 228(1), pp. 60-66.

[5] Adamczyk, P. G., and Kuo, A. D., 2015. "Mechanisms of gait asymmetry due to push-off deficiency in unilateral amputees". Neural Systems and Rehabilitation Engineering, IEEE Transactions on, 23(5), pp. 776-785.

[6] Major, M. J., Kenney, L. P., Twiste, M., and Howard, D., 2012. "Stance phase mechanical characterization of transtibial prostheses distal to the socket: a review". Jour- 
nal of Rehabilitation Research and Development, 49(6), pp. 815-829.

[7] Zelik, K. E., Collins, S. H., Adamczyk, P. G., Segal, A. D., Klute, G. K., Morgenroth, D. C., Hahn, M. E., Orendurff, M. S., Czerniecki, J. M., and Kuo, A. D., 2011. "Systematic variation of prosthetic foot spring affects center-ofmass mechanics and metabolic cost during walking". IEEE Transactions on Neural Systems and Rehabilitation Engineering, 19(4), pp. 411-419.

[8] Houdijk, H., Pollmann, E., Groenewold, M., Wiggerts, H., and Polomski, W., 2009. "The energy cost for the step-tostep transition in amputee walking". Gait \& posture, 30(1), pp. 35-40.

[9] Linde, V. D., Cheriel, J., and Alexander, C. H., 2004. "A systematic literature review of the effect of different prosthetic components on human functioning with a lower-limb prosthesis". Journal of Rehabilitation Research \& Development, 41(4), pp. 555-570.

[10] Hafner, B. J., 2005. "Clinical Prescription and Use of Prosthetic Foot and Ankle Mechanisms: A Review of the Literature". Journal of Prosthetics and Orthotics, 17(4), pp. S5S11.

[11] Hofstad, C., Van Der Linde, H., Van Limbeek, J., and Postema, K., 2009. "Prescription of prosthetic ankle-foot mechanisms after lower limb amputation ( Review )". The Cochrane Library(1).

[12] Fey, N. P., Klute, G. K., and Neptune, R. R., 2011. “The influence of energy storage and return foot stiffness on walking mechanics and muscle activity in below-knee amputees". Clinical Biomechanics, 26, pp. 1025-1032.

[13] Hansen, a. H., Childress, D. S., and Knox, E. H., 2000. "Prosthetic foot rollover shapes with implications for alignment of transtibial prostheses". Prosthetics and Orthotics International, 24(3), jan, pp. 205-215.

[14] Hansen, A. H., and Childress, D. S., 2005. "Effects of adding weight to the torso on roll-over characteristics of walking". The Journal of Rehabilitation Research and Development, 42(3), p. 381.

[15] Hansen, A. H., and Childress, D. S., 2004. "Effects of shoe heel height on biologic rollover characteristics during walking". Journal of Rehabilitation Research \& Development, 41(4), pp. 547-554.

[16] Hansen, A. H., Childress, D. S., and Knox, E. H., 2004. "Roll-over shapes of human locomotor systems: effects of walking speed". Clinical Biomechanics, 19, pp. 407-414.

[17] Olesnavage, K. M., and Winter, A. G. Correlating mechanical design of passive prosthetic feet to gait kinematics using a novel optimization parameter: lower leg trajectory error.

[18] Olesnavage, K. M., and Winter V., A. G., 2015. "Lower Leg Trajectory Error: A novel optimization parameter for designing passive prosthetic feet". In IEEE International Conference on Rehabilitation Robotics (ICORR), pp. 271-
276.

[19] Olesnavage, K. M., and Winter V., A. G., 2016. "Design and preliminary testing of a prototype for evaluating lower leg trajectory error as an optimization metric for prosthetic feet". In ASME International Design Engineering Technical Conferences and Computers and Information in Engineering Conference (IDETC/CIE).

[20] Olesnavage, K. M., and Winter V., A. G., 2015. "Design and qualitative testing of a prosthetic foot with rotational ankle and metatarsal joints to mimic physiological roll-over shape". In ASME International Design Engineering Technical Conferences and Computers and Information in Engineering Conference (IDETC/CIE).

[21] Winter, D. A., 2009. Biomechanics and Motor Control of Human Movement, 4th ed. John Wiley \& Sons, Inc., Hoboken, New Jersey.

[22] Howell, L. L., 2001. Compliant mechanisms. John Wiley \& Sons.

[23] Lyu, N., and Saitou, K., 2003. "Topology optimization of multi-component structures via decomposition-based assembly synthesis". In ASME 2003 International Design Engineering Technical Conferences and Computers and Information in Engineering Conference, American Society of Mechanical Engineers, pp. 269-281.

[24] Ananthasuresh, G., 2001. "Topology synthesis of compliant mechanisms for nonlinear force-deflection and curved path specifications".

[25] Lu, K.-J., and Kota, S., 2006. "Topology and dimensional synthesis of compliant mechanisms using discrete optimization”. Journal of Mechanical Design, 128(5), pp. 1080-1091.

[26] Bruns, T. E., and Tortorelli, D. A., 2001. "Topology optimization of non-linear elastic structures and compliant mechanisms". Computer Methods in Applied Mechanics and Engineering, 190(26), pp. 3443-3459.

[27] Santer, M., and Pellegrino, S., 2009. "Topological optimization of compliant adaptive wing structure". AIAA journal, 47(3), pp. 523-534.

[28] Kikuchi, N., and Kota, S., 1997. "Topological synthesis of compliant mechanisms using multi-criteria optimization".

[29] Cao, L., Dolovich, A. T., and Zhang, W. C., 2015. "Hybrid compliant mechanism design using a mixed mesh of flexure hinge elements and beam elements through topology optimization". Journal of Mechanical Design, 137(9), p. 092303.

[30] Deepak, S. R., Dinesh, M., Sahu, D. K., and Ananthasuresh, G., 2009. "A comparative study of the formulations and benchmark problems for the topology optimization of compliant mechanisms". Journal of Mechanisms and Robotics, 1(1), p. 011003.

[31] Xu, D., and Ananthasuresh, G., 2003. "Freeform skeletal shape optimization of compliant mechanisms". 
TRANSACTIONS-AMERICAN SOCIETY OF MECHANICAL ENGINEERS JOURNAL OF MECHANICAL DESIGN, 125(2), pp. 253-261.

[32] Lan, C.-C., and Cheng, Y.-J., 2008. "Distributed shape optimization of compliant mechanisms using intrinsic functions". Journal of Mechanical Design, 130(7), p. 072304.

[33] Hetrick, J., and Kota, S., 1999. "An energy formulation for parametric size and shape optimization of compliant mechanisms". Ann Arbor, 1050, p. 48109.

[34] Zhou, H., and Ting, K.-L., 2006. "Shape and size synthesis of compliant mechanisms using wide curve theory". Journal of Mechanical Design, 128(3), pp. 551-558. 